Both Siddiqui and Pedley and their colleagues caution that the polymorphism in the $\mathrm{ABCB} 1$ gene may not be the specific cause of drug resistance, and other associations may be uncovered in different populations. Pharmacogenomics opens a new approach to the development of therapeutic agents in epilepsy.

A loss of $\mathrm{Na}^{+}$channel drug sensitivity is proposed as a novel mechanism underlying drug, specifically carbamazepine (CBZ), refractory epilepsy (Remy S, Gabriel S, Urban BW, et al. Ann Neurol April 2003;53:469-479). Researchers at the University of Bonn Medical Center, and Charite, Berlin, Germany, studied cellular mechanisms underlying drug resistance in resected hippocampal tissue from patients with temporal lobe epilepsy. The mechanism of action of CBZ, use-dependent block of voltage-dependent $\mathrm{Na}^{+}$ channels, is completely lost in CBZ-resistant patients.

\title{
EEG IN PANAYIOTOPOULOS SYNDROME
}

The sequential changes in localization of EEG foci with age, and the relation between the clinical manifestations and EEG pattern in Panayiotopoulos syndrome (PS) were analyzed in a study of 76 children ( 37 boys and 39 girls) followed for $>2$ years at Tokyo Woman's Medical University, Japan. PS syndrome (early-onset benign occipital seizure susceptibility syndrome) is characterized by the following: onset of epilepsy between ages 1 and 8 years; attacks of ictal vomiting and eye deviations, with or without secondary generalization; normal development before seizure onset; normal neuroimaging; occipital EEG foci, at times shifting to centrotemporal or frontal regions with age; and remission before age 12 years. In this study, the EEG findings were excluded from the criteria for inclusion. A history of febrile convulsions was recorded in $33(43.4 \%)$ patients, and a family history of seizure disorder in $21(27.6 \%)$. The age at onset of epilepsy ranged from 14 to 118 months (average 48 months). Seizures recurred from 0 to 139 months (median 70 months); the total number in each case was 1 to 27 (mean 4.8). Status epilepticus occurred in $45(61.6 \%)$ cases.

The EEG occipital spike focus ( 12 cases) was seen most frequently at 2 to 5 years of age; independent and synchronous frontopolar and occipital spikes (Fp-O spikes) (14 cases) at 4 to 7 years: and centroparietotemporal (CPT) spike foci (21 cases) at 6 to 10 years. EEG foci showed frequent shifting, multiplications, and generalization with age. The Fp-O group showed the latest age at onset of epilepsy. The generalized EEG pattern (19 cases) had the highest frequency of seizures and status epilepticus, and the longest active seizure period. The prognosis was favorable regardless of the EEG pattern. (Ohtsu $\mathrm{M}$, Oguni $\mathrm{H}$, Hayashi $\mathrm{K}$, et al. EEG in children with early-onset benign occipital seizure susceptibility syndrome: Panayiotopoulos syndrome. Epilepsia 2003;44:435-442). (Reprints: Dr H Oguni, Department of Pediatrics, Tokyo Woman's Medical University, 81 Kawada-cho, Shinjuku-ku, Tokyo 162, Japan).

COMMENT. EEG foci in Panayiotopoulos (PS) syndrome do not persistently localize to occipital regions but tend to shift location, multiply, and become generalized with age. The various EEG patterns tend to appear at different ages and to be associated with certain clinical characteristics. For example, the generalized EEG pattern group has the highest frequency of seizures, status epilepticus (SE), and longest active 
seizure period. In contrast, the CPT group has the shortest active seizure period, but often presents with SE. The most characteristic clinical manifestation, occurring in all patients, is the ictal vomiting. In PS a genetic predisposition to seizures is common. The outlook is generally good, with seizure remission before 12 years of age.

Abnormal neuroimaging in patients with BECTS is reported in $15 \%$ of 71 cases (Gelisse P, Corda D, Raybaud C, et al. Epilepsia 2003;44:372-378). These included dilated lateral ventricles, neonatal intraventricular hemorrhage, and agenesis of corpus callosum.

\section{EFFECT OF METHYLPHENIDATE IN ADHD WITH EPILEPSY OR WITH EEG ABNORMALITIES WITHOUT SEIZURES}

The safety and efficacy of methylphenidate (MPH) in the treatment of ADHD, group 1) with epilepsy and group 2) with EEG abnormalities without seizures, were determined in a study of 119 children ( 98 males, 21 females; ages 6 to 16 years) at Gazi University, Ankara, Turkey. The neurologic examination showed only soft signs in $8 \%$ of patients. Brain scans, vision and hearing were normal, and WISC-R scores were average (mean $92.3+/-0.2$ ). Of 57 patients with epilepsy, 34 had complex partial seizures, $13 \mathrm{had}$ tonic-clonic seizures, and 10 had multiple seizures; 52 received antiepileptic (AED) monotherapy and 5 polytherapy. At the end of the 12 month period of AED with added MPH $(0.3-1 \mathrm{mg} / \mathrm{kg} /$ day $)$ treatment, the mean yearly seizure frequency was unchanged $(8.2$ pre-MPH treatment and 8.1 post-treatment), but $5(10 \%)$ patients had an increase in seizures. None of the 62 patients with ADHD and EEG abnormalities developed seizures during treatment with MPH (without AED). Percentage of patients with EEG epileptiform activity was decreased in both the ADHD+epilepsy group and the ADHD without seizure group; $35.1 \%$ at baseline of $19.3 \%$ post-treatment $(\mathrm{p}=0.01)$ and $24.2 \% \mathrm{cf} 12.9 \%(\mathrm{p}=0.02)$, respectively. The baseline and the last mean total ADHD and Conners' scores from parents' and teachers' questionnaires showed significant improvements in both the epileptic and the abnormal EEG groups. On clinician ratings, 92 of the 119 children in the study showed reduced ADHD symptoms, and only 9 patients had deterioration in behavior, 6 of whom were epileptic. Side effects of MPH were observed in $26(22 \%)$ patients and included loss of appetite (23), sleep disorder (19), stomachache (14), headache (14), and motor tics (2); none required MPH withdrawal. MPH is considered safe and effective in patients with ADHD and epilepsy, when combined with AED treatment, and in patients with ADHD and abnormal EEG without seizures. (Gucuyener K, Erdemoglu AK, Senoi S, et al. Use of methylphenidate for attention-deficit hyperactivity disorder in patients with epilepsy or electroencephalographic abnormalities. J Child Neurol 2003;18:100-112). (Dr A Kemal Erdemoglu, Cinnah Cad Kirkpinar sok 12/4 Cankaya, Ankara 06690, Turkey).

COMMENT. The PDR advises against the use of methylphenidate (MPH) in patients with seizures or with abnormal EEG. MPH is thought to lower the convulsive threshold. The present report confirms that of previous authors, and shows that MPH may be a safe and effective treatment for ADHD complicated by epilepsy, provided that seizures are controlled by anticonvulsant medication (In Millichap JG. Attention Deficit Hyperactivity and Learning Disorders. PNB Publishers, 1998;pp179-181). MPH should be discontinued or the dosage reduced, if seizures recur despite optimal doses of AED. The 